\title{
Partition Function Formalism in the Problem of Multidimensional Integer Partitions
}

\author{
Andrij Rovenchak \\ Department for Theoretical Physics, \\ Ivan Franko National University of Lviv, \\ 12 Drahomanov St., Lviv, UA-79005, Ukraine \\ e-mail: andrij.rovenchak@gmail.com
}

(Received: 23 March 2010; accepted: 1 July 2010; published online: 25 August 2010)

\begin{abstract}
The formalism based on the microcanonical treatment of a many-boson system is applied to the problem in the number theory known as the partitioning of an integer. An estimation is obtained for the asymptotic behavior of the number of multidimensional partitions into sums of various powers of integers. The obtained results are shown to reproduce the known ones for linear summands.

Key words: integer partitions, multimensional partition, bosonic systems
\end{abstract}

\section{INTRODUCTION}

Partitioning of integers is a problem in the number theory dating back to Leibniz [1] and Euler [2]. A partition of a positive integer $n$ is a way of writing $n$ as a sum of positive integers, where the order of the summands is not significant. The number of partitions is called the partition function and is designated as $p(n)[3$, Ch. 1]. To avoid confusion with the respective physical term, further we refer to $p(n)$ simply as the number of partitions.

Consider number 5 . It can be represented as follows:

$$
\begin{gathered}
5=4+1=3+2=3+1+1=2+2+1= \\
=2+1+1+1=1+1+1+1+1 .
\end{gathered}
$$

Thus, the number of partitions $p(5)=7$.

If a positive integer $n$ is written as a sum on $s$-th powers of positive integers, e.g.,

$$
5=2^{2}+1^{2}=1^{2}+1^{2}+1^{2}+1^{2}+1^{2},
$$

a generalization of the previously defined $p(n)$ function is $p^{s}(n): p^{2}(5)=2$.

$$
p(n)=p^{1}(n) .
$$

While the sequence of summands is not significant (their permutation does not produce a new partition), they are conventionally written in a non-increasing order, as shown in the above examples.

When defining higher-dimensional partitions, the fact that sequences are non-increasing in all the directions becomes important. In the $D$-dimensional case, an integer $n$ is represented as a sum of positive integers $n_{i_{1} \ldots i_{D}}$ :

$$
n=\sum_{i_{1}, \ldots i_{D} \geq 0} n_{i_{1} \ldots i_{D}}
$$

where $n_{i_{1} \ldots i_{D}} \geq n_{j_{1} \ldots j_{D}}$ whenever $i_{1} \leq j_{1}, i_{2} \leq j_{2}, \ldots, i_{D} \leq j_{D}[3$, p. 179].

Consider two-dimensional (plane) partitions of 3

$$
\begin{array}{rrrrr}
32+1 & 2 & 1+1+1 & 1+1 & 1 \\
& + & + & + \\
& 1 & & 1 \\
& & & + \\
& & & \\
& & & &
\end{array}
$$

and note the ordering in both left-to-right and top-tobottom directions $[3$, Ch. $11 ; 4]$. 


\section{PHYSICAL ANALOGY}

The number of partitions has a clear physical analogy with the number of microstates of a many-boson system [5-7]. Indeed, the number of microstates $\Gamma(E)$ is the number of ways to distribute the energy $E$ over the indistinguishable (and thus quantum, not classical) particles. The latter ensures that the order of summands is not significant. As a partition can have any number of equal summands, the bosonic system is an appropriate one (fermions can be used to analyze the so called distinct partitions).

To illustrate the above statement, consider for simplicity a system of one-dimensional harmonic oscillators with single-particle energy levels given by $\varepsilon_{m}=\hbar \omega m$. Physically, this system corresponds to bosons trapped in a highly asymmetric harmonic trap. Let the energy to be distributed over the particles equal $E=\hbar \omega n$. The number of microstates $\Gamma(E)$ is defined by the number of partitions of $n$, for which an asymptotic expression is given by the well-known Hardy-Ramanujan formula [8]:

$$
p(n)=\frac{1}{4 \sqrt{3} n} e^{\pi \sqrt{2 / 3} \sqrt{n}}
$$

and hence

$$
\Gamma(E)=\frac{1}{4 \sqrt{3} E / \hbar \omega} e^{\pi \sqrt{2 / 3} \sqrt{E / \hbar \omega}} .
$$

The extension to higher dimensions is not straightforward, however. For instance, the two-dimensional partitions demonstrated in (2) correspond to a simple spectrum $\varepsilon_{m_{1}, m_{2}}=m_{1}+m_{2}$. The energies of the particles reproducing the partitions are (reading left to right): $\left\{\varepsilon_{30}\right\},\left\{\varepsilon_{20}, \varepsilon_{10}\right\},\left\{\varepsilon_{20}, \varepsilon_{01}\right\},\left\{\varepsilon_{10}, \varepsilon_{10}, \varepsilon_{10}\right\},\left\{\varepsilon_{10}, \varepsilon_{10}, \varepsilon_{01}\right\}$, and $\left\{\varepsilon_{10}, \varepsilon_{01}, \varepsilon_{01}\right\}$. Clearly, some states are missing, like $\left\{\varepsilon_{03}\right\}$, $\left\{\varepsilon_{10}, \varepsilon_{02}\right\}$, etc. Therefore, applying such a physical analogy we slightly overestimate the number of partitions, but this does not influence the leading asymptotics as shown below.

\section{POWER ENERGY SPECTRUM}

We consider the energy spectrum of a $D$-dimensional system in the following form:

$$
\varepsilon\left(m_{1}, \ldots, m_{D}\right)=m_{1}^{s_{1}}+\ldots+m_{D}^{s_{D}} .
$$

In fact, only $s_{i}=1$ and $s_{i}=2$ cases are physically realistic [9], but other values can effectively occur in some exotic model systems or in the density of states of a system confined by an external potential within a WKB approach. Having different powers corresponds to a special geometry of a system under consideration, e. g. free particles moving along $z$ axis $\left(s_{3}=2\right)$ confined by a two-dimensional harmonic trap in $x$ and $y$ directions $\left(s_{1}=s_{2}=1\right)$.

To obtain $\Gamma(E)$ we follow the derivation given in [6], see also [10].

Partition function $Z(\beta)$ and the number of microstates $\Gamma(E)$ are related via the Laplace transform:

$$
\begin{aligned}
& Z(\beta)=\int_{0}^{\infty} \Gamma(E)^{-\beta E} d E, \\
& \Gamma(E)=\frac{1}{2 \pi i} \int_{-i \infty}^{+i \infty} Z(\beta) e^{\beta E} d \beta .
\end{aligned}
$$

The entropy $S(\beta)$ equals

$$
S(\beta)=\beta E+\ln Z(\beta) .
$$

For energy spectrum (5) the partition function is

$$
\begin{aligned}
& Z(\beta)=\prod_{\text {all the energies }}\left(1-e^{-\beta \varepsilon}\right)^{-1}= \\
& =\prod_{m_{1}=1}^{\infty} \ldots \prod_{m_{D}=D}^{\infty}\left[1-e^{-\beta\left(m_{1}^{s_{1}}+\ldots+m_{D}^{s_{D}}\right)}\right]^{-1} .
\end{aligned}
$$

Using the saddle-point method, one can evaluate $\Gamma(E)$ from Eq. (6) as follows:

$$
\Gamma(E)=\frac{\exp \left[S\left(\beta_{0}\right)\right]}{\sqrt{2 \pi S^{\prime \prime}\left(\beta_{0}\right)}} .
$$

The entropy $S(\beta)$, after applying the Euler-Maclaurin summation formula, can be expressed in such a form

$$
\begin{gathered}
S(\beta)=\beta E-\sum_{m_{1}=1}^{\infty} \ldots \sum_{m_{D}=1}^{\infty} \ln \left[1-e^{-\beta\left(m_{1}^{s_{1}}+\ldots+m_{D}^{s_{D}}\right)}\right]= \\
=\beta E+\frac{C}{\beta^{1 / \sigma}}+\frac{1}{2^{D}} \ln \beta+\ldots,
\end{gathered}
$$

where the last transformation is performed in the limit of $\beta \rightarrow 0$. Here, $C=C\left(s_{1}, \ldots, s_{D}\right)$ is a constant with a complex dependence on the powers $s_{1}, \ldots, s_{D}$. For our estimations, its explicit form is not required. The parameter $\sigma$ is defined by

$$
\frac{1}{\sigma}=\frac{1}{s_{1}}+\ldots+\frac{1}{s_{D}} .
$$

The coefficient $1 / 2^{D}$ comes from the $D$-dimensional generalization of the summation formula due to a rectangular nature of the summation domain, cf. [11, p. 892]: only the lowermost nod $m_{1}=\ldots=m_{D}=1$ contributes as the first correction to the integral term. 
For the stationary point $\beta_{0}, S^{\prime}\left(\beta_{0}\right)=0$, one obtains

$$
\beta_{0}=\left(\frac{C}{\sigma E}\right)^{\sigma /(\sigma+1)}=\lambda E^{-\sigma /(\sigma+1)} \text {. }
$$

Thus, the number of microstates is

$$
\left.\Gamma(E) \sim E^{-\frac{1}{\sigma+1}\left(\frac{1}{2}+\left(2^{-D}+1\right) \sigma\right.}\right) \exp \left[\lambda(\sigma+1) E^{\frac{1}{\sigma+1}}\right] .
$$

Substituting $E$ with $n$, one obtains the expression for the number of partitions

$$
\begin{gathered}
p\left(s_{1}, \ldots, s_{D} ; D \mid n\right) \sim \\
\sim n^{-\frac{1}{\sigma+1}\left(\frac{1}{2}+\left(2^{-D}+1\right) \sigma\right)} \exp \left[\lambda(\sigma+1) n^{\left.\frac{1}{\sigma+1}\right]},\right. \\
\sigma=\left(\frac{1}{s_{1}}+\ldots+\frac{1}{s_{D}}\right)^{(-1)} .
\end{gathered}
$$

The leading asymptotic behavior of the function $p\left(s_{1}\right.$, $\left.\ldots, s_{D} ; D \mid n\right)$ is thus entirely governed by the parameter $\sigma$. If all $s_{i}$ 's are equal, $s_{1}=\ldots=s_{D}=s$.

$$
\sigma=s / D \text {. }
$$

In particular, for simple multidimensional partitions $(s=1)$ we obtain:

$$
p(D \mid n) \sim n^{-\frac{1}{D+1}\left(\frac{D}{2}+2^{-D}+1\right)} \exp \left[\operatorname{const} n^{\frac{D}{D+1}}\right] .
$$

\section{DISCUSSION}

For multidimensional partitions, Eq. (15) reproduces the known leading asymptotics in the exponential correctly [12]. Plane partitions corresponding to $D=2$ are studied in more detail, and it is worth making the comparison in this special case.

One has from Eq. (15)

$$
p(2 \mid n) \sim n^{-3 / 4} \exp \left(\text { const } n^{2 / 3}\right) .
$$

A correct asymptotics, according to Wright (1931) is known to be [4]

$$
p(2 \mid n) \sim n^{-25 / 36} \exp \left(\text { const } n^{2 / 3}\right),
$$

and differs from our result only in the pre-exponential factor. Expression (16) gives a close estimate for the number of plane partitions,

$$
n^{-3 / 4}=n^{-0.75 \ldots} \quad \text { while } \quad n^{-25 / 36}=n^{-0.694 \ldots} .
$$

For the so called solid partitions $(D=3)$ [13] the estimation (15) is also correct, at least in the leading order.

In summary, the expression for the number of multidimensional partitions of an integer is derived in a general case of summands with different powers. The obtained estimation is shown to coincide in the leading order with known asymptotic results.

\section{References}

[1] G.W. Leibniz, Specimen de divulsionibus aequationum ad problemata indefinita in numeris rationalibus solvenda. 2. September 1674, in: G.W. Leibniz, Sämtliche Schriften und Briefe. Siebente Reihe: Mathematische Schriften, Bd. 1: 1672-1676. Geometrie - ahlentheorie - Algebra (1. Teil), Akademie-Verlag, Berlin, 740-755 (1990).

[2] L. Eulero, De partitione numerorum. Novi Commentarii Academiae scientiarum Petropolitanae 3, 125-169 (1753).

[3] G.E. Andrews, The Theory of Partitions. Addison-Wesley, Reading, Mass. (1976).

[4] G. Almkvist, Asymptotic formulas and generalized Dedekind sums. Experimental Mathematics 7, 343-359 (1998).

[5] S. Grossmann, M. Holthaus, Fluctuations of the particle number in a trapped Bose-Einstein condensate. Phys. Rev. Lett. 79, 3557-3560 (1997).

[6] M.N. Tran, M.V.N. Murthy, R.J. Bhaduri, On the quantum density of states and partitioning an integer. Ann. Phys. 311, 204-219 (2004).

[7] A. Comtet, P. Leboeuf, S.N. Majumdar, Level density of a Bose gas and extreme value statistics. Phys. Rev. Lett. 98, 070404 (4 p.) (2007).

[8] G.H. Hardy, S. Ramanujan, Asymptotic formulae in combinatory analysis, Proc. London Math. Soc. 17, 75-115 (1918).

[9] C.S. Srivatsan, M.V.N. Murthy, R.K. Bhaduri, Gentile statistics and restricted partitions, Pramana - J. Phys. 66, 485-494 (2006).

[10] A. Rovenchak, The relation between fractional statistics and finite bosonic systems in one-dimensional case, Fiz. Nizk. Temp. 35, 510-513 (2009); Low Temp. Phys. 35, 400-403 (2009).

[11] M. Abramowitz, I.A. Stegun, Handbook of mathematical functions. Tenth printing. National Bureau of Standards 1972.

[12] D.P. Bhatia, M.A. Prasad, D. Arora, Asymptotic results for the number of multidimensional partitions of an integer and directed compact lattice animals. J. Phys. A: Math. Gen. 30, 2281-2285 (1997).

[13] V. Mustonen, R. Rajesh, Numerical estimation of the asymptotic behaviour of solid partitions of an integer. J. Phys. A: Math. Gen. 36, 6651-6659 (2003). 


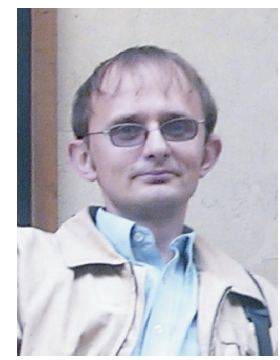

AndRIJ Rovenchak graduated in Physics in 1998. He received the PhD degree in Theoretical Physics from the Ivan Franko National University of Lviv (2003). He works as an Associate Professor at the Department for Theoretical Physics, National University of Lviv. His research interests concern quantum liquids, bosonic systems, fractional statistics, as well as history of science and quantitative methods in linguistics. 